\title{
TE Surface Waves in a Plasma Sandwich Structure
}

\author{
M.N. Shaikh, B. ZAMIR AND R. Ali* \\ Department of Physics, University of the Punjab, Quaid-i-Azam Campus, Lahore-54590, Pakistan \\ (Received May 15, 2014; revised version March 7, 2015; in final form April 2, 2015)
}

\begin{abstract}
Transverse electric surface waves are analyzed in a plasma medium bounded by a left-handed material. A dispersion relation is obtained by applying the boundary conditions for the tangential field components. Numerical investigations show the dependence of effective wave index on the propagation frequency for different thicknesses and number densities of the plasma medium and also for different values of the filling factor of left-handed material.
\end{abstract}

DOI: $10.12693 /$ APhysPolA.127.1625

PACS: 41.20.Jb, 84.40.Az

\section{Introduction}

The analyses of parallel-plate, sandwiched and slab waveguide structures exhibit many interesting features in the research of microwave devices [1-8]. The practical implications of these studies are very important in microwave research when applied to optical and communication engineering, etc. In these structures, various electromagnetic and magnetostatic modes have been investigated and many advances in theoretical and numerical analysis have been published. Over the last few decades, much attention has been paid to the waveguide structures consisting of left-handed materials (LHMs) i.e. the materials with negative effective permittivity and permeability in a certain frequency range and as a consequence differs substantially in the propagation properties from conventional dielectric media [9-18]. For example, in a symmetric slab waveguide structure the dispersion characteristics of electromagnetic surface waves of a ferrite slab bounded by LHMs have been investigated by ElKhozondar et al. [5]. Their results show that surface waves in the microwave region can only be satisfied under the condition of negative effective permeability of the ferrite slab. Properties of magnetostatic surface waves have been investigated by $\mathrm{Ma}$ et al. [4] in a magnetic structure with one LHM film sandwiched between two ferromagnetic films. They showed the possibility of manufacturing a sandwich structure over some frequency range and on modeling these structures so that they may be used to control the dispersive effects of materials. In another symmetric slab waveguide structure constructed from $\mathrm{SiO}_{2}$ dielectric guiding core sandwiched between lossy LHMs, Ashour [18] derived the modal dispersion relation for transverse electric (TE) modes. He showed that the waveguide structure is a good candidate for coupling or guiding of electromagnetic waves. Similarly, guided modes have been studied in asymmetric LHM slab waveguide structures. For example, He et al. [19] and Wang and Dong [20] investigated the dispersive characteristics of the metal-LHM-air and dielectric-LHM-dielectric asymmetric waveguide structures, respectively.

*corresponding author; e-mail: rashid.physics@pu.edu.pk
The research of plasma-filled waveguide structures is motivated by small-sized electronic devices, which are able to operate in the microwave frequency band and are continuously tunable over a broad frequency range (see, for example [21] and references therein). In the waveguide structures, a plasma filling strongly effects the dispersion properties of the different wave modes. Interest has been shown in studying plasma-filled waveguides in the past. For example, Mukherjee [22] investigated TE modes in a plane-parallel waveguide filled with warm plasma and obtained the dispersion relation and the cutoff frequencies. He has also presented numerical results of the cutoff frequencies for different parameters characterizing electron-plasma temperature, velocity and the applied magnetic field. Ghosh and Pal [1] investigated the dispersion relations of $\mathrm{TE}$ and transverse magnetic (TM) modes in a plasma-filled parallel plate waveguide with one boundary corrugated. In another paper with a similar geometry, Prasad et al. [2] investigated the relativistic effects on the TM mode and found that the effect of temperature is remarkably insignificant unlike for the non-relativistic case. Plasma-filled cylindrical waveguides have also been investigated in the research of communication devices, e.g. Assis and Sakanaka [23] studied electromagnetic waves propagation in a bounded magnetized and warm plasma. In recent years, a lot of effort has been made to study different physical and geometrical parameters for the wave propagation in plasma-filled waveguides (see e.g. Khalil and Mousa [24] and references therein).

Since most of the waveguides structures used in the research of wave propagation in the microwave region of communication devices include LHMs (e.g. [3-5]). We are thus motivated to explore a symmetric slab waveguide structure consisting of a plasma medium sandwiched between lossless LHMs. This work may be useful for potential applications in the designing of antennae, microstrips, and waveguide couplers [16-18]. The features of our proposed structure may provide exciting ideas for the designing of other waveguide structures, with possible applications in this area of research.

In this work, we discuss the dispersion properties of TE surface waves in the plasma medium bounded by a LHM. This paper is organized as follows. In Sect. 2, 
mathematical formulation for the proposed structure is presented. Section 3 is devoted to the derivation of the dispersion relation. Results and discussion are presented in Sect. 4, where we plot and discuss the effective wave index versus propagation frequency. The conclusion is presented in Sect. 5.

\section{Mathematical formulation}

The geometry of the sandwich structure for the propagation of TE surface waves to be analyzed is shown in Fig. 1. We consider a plasma medium $(0 \leq x \leq d)$

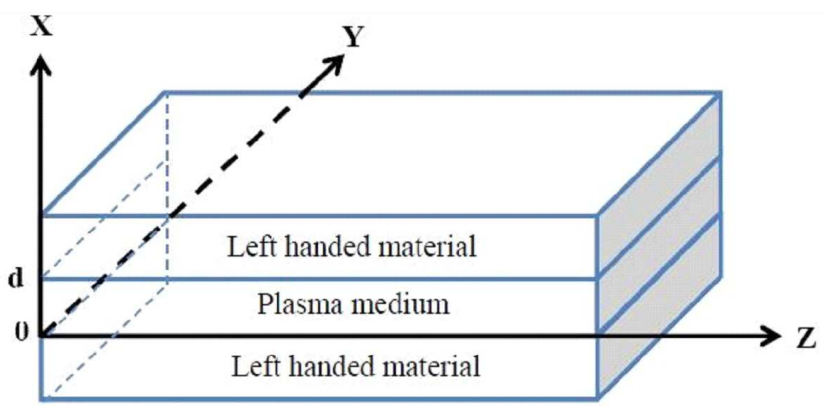

Fig. 1. A schematic representation of LHM/plasma/LHM structure.

bounded by LHM at $(x<0)$ and $(d<x)$. For simplicity, we assume that the propagation of these TE surface waves are in the $+z$ direction and that all field components are proportional to $\mathrm{e}^{\mathrm{i}(k z-\omega t)}$ which are independent of $y$ (i.e. $\partial / \partial y=0$ ), where $k$ is the wave number in the $z$ direction and $\omega$ is the propagation frequency. In the present geometry, TE field components are taken as

$$
E=\left(0, E_{y}, 0\right) \mathrm{e}^{\mathrm{i}(k z-\omega t)}, \quad H=\left(H_{x}, 0, H_{z}\right) \mathrm{e}^{\mathrm{i}(k z-\omega t)} .
$$

\subsection{Calculations for the plasma medium $(0 \leq x \leq d)$}

For the plasma like medium, using time dependent perturbations, the following linearized equations are used to describe the plasma motion in the absence of an equilibrium electron drift velocity and electric field [1, 23, 25]:

$$
\begin{aligned}
& \mathrm{i} \omega p=n_{0} m U^{2} \boldsymbol{\nabla} \cdot \boldsymbol{V}, \\
& n_{0} m(-\mathrm{i} \omega \boldsymbol{V}+\nu \boldsymbol{V})=-e n_{0} \boldsymbol{E}-\nabla p, \\
& \boldsymbol{\nabla} \times \boldsymbol{H}=-e n_{0} \boldsymbol{V}-\mathrm{i} \omega \varepsilon_{0} \boldsymbol{E}, \\
& \boldsymbol{\nabla} \times \boldsymbol{E}=\mathrm{i} \omega \mu_{0} \boldsymbol{H} .
\end{aligned}
$$

In the above set of equations $m, \boldsymbol{V}, p, n_{0}, \nu$, and $U=(\gamma K T / m)^{1 / 2}$ are the mass, velocity, pressure, number density, collision frequency, and thermal velocity of the electrons, respectively, whereas $\dot{\gamma}, K$ and $T$ are ratio of specific heats, the Boltzmann constant, and temperature of the plasma. Eliminating velocity $\boldsymbol{V}$ from Eq. (3) by using Eqs. (1) and (2) we get

$$
\boldsymbol{\nabla} \times \boldsymbol{H}=-\mathrm{i} \omega \varepsilon_{0} \varepsilon_{p} \boldsymbol{E}-\frac{\mathrm{i} \omega \varepsilon_{0} U^{2}}{\omega^{2}+\mathrm{i} v \omega} \nabla(\nabla \cdot \boldsymbol{E}),
$$

where

$$
\varepsilon_{\mathrm{p}}=1-\frac{\omega_{\mathrm{p}}^{2}}{\omega^{2}+\mathrm{i} v \omega},
$$

$\omega_{\mathrm{p}}$ is the electron plasma frequency and is given by $\omega_{\mathrm{p}}=\left(e^{2} n_{0} / \varepsilon_{0} m\right)^{1 / 2}$. Equations (4) and (5) give

$$
\begin{aligned}
& \boldsymbol{\nabla}(\boldsymbol{\nabla} \cdot \boldsymbol{E})-\nabla^{2} \boldsymbol{E}= \\
& \quad \mathrm{i} \omega \mu_{0}\left[-\mathrm{i} \omega \varepsilon_{0} \varepsilon_{\mathrm{p}} \boldsymbol{E}-\frac{\mathrm{i} \omega \varepsilon_{0} U^{2}}{\omega^{2}+\mathrm{i} v \omega} \nabla(\boldsymbol{\nabla} \cdot \boldsymbol{E})\right] .
\end{aligned}
$$

Since in the present geometry of TE surface waves we have taken $\boldsymbol{E}=E_{y} \hat{\boldsymbol{j}}$ and $\partial / \partial y=0$, this yields

$$
\boldsymbol{\nabla} \cdot \boldsymbol{E}=(\hat{\boldsymbol{i}} \partial / \partial x+\hat{\boldsymbol{k}} \partial / \partial z) \cdot\left(E_{y} \hat{\boldsymbol{j}}\right)=0 .
$$

Thus Eq. (7) reduces to

$$
\frac{\mathrm{d}^{2} E_{y}}{\mathrm{~d} x^{2}}-k_{\mathrm{p}}^{2} E_{y}=0,
$$

where $k_{\mathrm{p}}^{2}=k^{2}-k_{0}^{2} \varepsilon_{\mathrm{p}}$ and $k_{0}^{2}=\omega^{2} / c^{2}$. For Eq. (8), subsequently we can write the following surface wave solution:

$$
E_{y}=A \cosh \left(k_{\mathrm{p}} x\right)+B \sinh \left(k_{\mathrm{p}} x\right),
$$

The corresponding magnetic field components are obtained from Maxwell Eq. (4):

$$
\begin{aligned}
& H_{x}=\frac{-k}{\omega \mu_{0}}\left[A \cosh \left(k_{\mathrm{p}} x\right)+B \sinh \left(k_{\mathrm{p}} x\right)\right], \\
& H_{z}=\frac{-\mathrm{i} k_{\mathrm{p}}}{\omega \mu_{0}}\left[A \sinh \left(k_{\mathrm{p}} x\right)+B \cosh \left(k_{\mathrm{p}} x\right)\right] .
\end{aligned}
$$

2.2. Calculations for the LHM $(x<0$ and $d<x)$

Here we carry out similar calculations as were done in the previous section, but now for the LHM. The Maxwell field equations for the LHM are

$$
\boldsymbol{\nabla} \times \boldsymbol{H}=-\mathrm{i} \omega \varepsilon_{0} \varepsilon(\omega) \boldsymbol{E},
$$

$$
\nabla \times \boldsymbol{E}=\mathrm{i} \omega \mu_{0} \mu(\omega) \boldsymbol{H},
$$

The dielectric constant and relative magnetic permeability of the LHM are given by

$$
\varepsilon(\omega)=1-\frac{\omega_{\mathrm{pL}}^{2}}{\omega^{2}},
$$

and

$$
\mu(\omega)=1-\frac{F \omega^{2}}{\omega^{2}-\omega_{\mathrm{r}}^{2}},
$$

where $\omega_{\mathrm{pL}}$ is the plasma frequency for the LHM, $F$ is the filling factor of the split ring resonator, $\omega_{\mathrm{r}}$ is the resonance frequency $[3,26-30]$. Here the losses have been neglected and consequently from Eqs. (12) and (13), we obtain

$$
\frac{\mathrm{d}^{2} E_{y}}{\mathrm{~d} x^{2}}-k_{\mathrm{L}}^{2} E_{y}=0,
$$

where $k_{\mathrm{L}}^{2}=k^{2}-k_{0}^{2} \varepsilon(\omega) \mu(\omega)$. We write the surface wave solution in the following form:

$$
E_{y}=C \mathrm{e}^{k_{\mathrm{L}} x}+D \mathrm{e}^{-k_{\mathrm{L}} x} \text {. }
$$

The field components of TE surface waves for the LHM 


$$
\text { at } \begin{aligned}
x<0 & \text { are } \\
E_{y} & =C \mathrm{e}^{k_{\mathrm{L}} x}, \\
H_{x} & =\frac{-k}{\omega \mu_{0} \mu(\omega)} C \mathrm{e}^{k_{\mathrm{L}} x}, \\
H_{z} & =\frac{-\mathrm{i} k_{\mathrm{L}}}{\omega \mu_{0} \mu(\omega)} C \mathrm{e}^{k_{\mathrm{L}} x} .
\end{aligned}
$$

Here we consider that the field penetration length in LHM layer is much shorter than their thickness. Similarly, we can obtain the field components of TE surface waves for the LHM at $x>d$ as

$$
\begin{aligned}
& E_{y}=D \mathrm{e}^{-k_{\mathrm{L}} x}, \\
& H_{x}=\frac{-k}{\omega \mu_{0} \mu(\omega)} D \mathrm{e}^{-k_{\mathrm{L}} x}, \\
& H_{z}=\frac{\mathrm{i} k_{\mathrm{L}}}{\omega \mu_{0} \mu(\omega)} D \mathrm{e}^{-k_{\mathrm{L}} x} .
\end{aligned}
$$

\section{Dispersion relation}

The dispersion relation for TE surface waves can be obtained by applying the boundary conditions for the continuity of the tangential components of the electric and magnetic field vectors. For this purpose, continuity of the tangential components of electric field $E_{y}$ and magnetic field $H_{z}$ at $x=0$ and $x=d$ gives the following dispersion relation for the TE surface waves:

$$
\tanh \left(k_{\mathrm{p}} d\right)=\frac{-2 k_{\mathrm{p}} k_{\mathrm{L}} \mu(\omega)}{k_{\mathrm{L}}^{2}+k_{\mathrm{p}}^{2} \mu^{2}(\omega)} .
$$

The above dispersion relation (24) can be solved numerically for the TE surface waves propagating in the LHM/plasma/LHM structure. For this purpose, we use expressions of $k_{\mathrm{p}}$ and $k_{\mathrm{L}}$ as indicated in Eqs. (8) and (16), respectively and establish a relationship between the effective wave index $\beta=c k / \omega$ and the propagation frequency $\omega$ in the following form:

$$
\begin{aligned}
& \tanh \left(\frac{\omega d}{c}\left(\beta^{2}-\varepsilon_{\mathrm{p}}\right)^{\frac{1}{2}}\right)= \\
& \quad \frac{-2 \mu(\omega)\left[\beta^{2}-\varepsilon_{\mathrm{p}}\right]^{\frac{1}{2}}\left[\beta^{2}-\varepsilon(\omega) \mu(\omega)\right]^{\frac{1}{2}}}{\left[\beta^{2}-\varepsilon(\omega) \mu(\omega)\right]+\mu^{2}(\omega)\left[\beta^{2}-\varepsilon_{\mathrm{p}}\right]} .
\end{aligned}
$$

To analyze the dispersion characteristics, the expressions for $\varepsilon_{\mathrm{p}}, \varepsilon(\omega)$ and $\mu(\omega)$ can be used from Eqs. (6), (14) and (15), respectively.

\section{Results and discussion}

In this section, we numerically investigate the dispersion Eq. (25) for a LHM/plasma/LHM sandwiched structure. In this connection, we discuss the dependence of effective wave index $\beta$ on the propagation frequency $\omega$ for different values of thickness $d$ and number density $n_{0}$ of the plasma medium. Similarly, we also show a plot for the different values of the filling factor $F$ of the LHM. In the present analysis, we have assumed that $\nu / \omega \ll 1[1]$.
Figure 2a shows the dependence of effective wave in$\operatorname{dex} \beta$ on the propagation frequency $\omega$ for different values of the thickness $d$ of the plasma medium i.e. $d=10^{-4} \mathrm{~m}$, $d=0.5 \times 10^{-4} \mathrm{~m}$ and $d=0.1 \times 10^{-4} \mathrm{~m}$. The parameters values chosen for the LHM are $\omega_{\mathrm{pL}}=10^{10} \mathrm{~Hz}$, $\omega_{\mathrm{r}}=4 \times 10^{9} \mathrm{~Hz}$ and $F=0.56$. For these numerical values, the frequency band extends from $4 \times 10^{9} \mathrm{~Hz}$ to $6 \times 10^{9} \mathrm{~Hz}$, for which the permittivity and permeability of LHM have negative values simultaneously [3, 28, 30]. For the plasma medium, we consider typical values of electron number density $n_{0}$ for an over-dense plasma regime with $\varepsilon_{\mathrm{p}}=1-\omega_{\mathrm{p}}^{2} / \omega^{2}<0$ when $\omega<\omega_{\mathrm{p}}$. Thus for $n_{0}=10^{17} \mathrm{~m}^{-3}$ Fig. 2a shows that for each thickness $d$ of the plasma medium, there are two propagation regions around a gap (or region of non-propagation) within the frequency band from $4 \times 10^{9} \mathrm{~Hz}$ to $6 \times 10^{9} \mathrm{~Hz}$. We observe that with the increase of effective wave index $\beta$, the propagation gap decreases. To show this trend we have chosen large values of $\beta$. It may be noted that in Refs. [5, 31] much higher values of $\beta$ have been used to show such trends. It is observed that in the upper region of propagation the frequency $\omega$ shows a decrease with the increase of effective wave index $\beta$. Since a dispersion curve's slope gives the group velocity, thus a negative slope means that backward wave propagation occurs and our proposed structure in the upper region of propagation behaves like a LHM [28], whereas the lower region of propagation shows an opposite trend. It should further be noted that as the thickness $d$ of plasma medium decreases, the propagation gap increases. This shows that propagation characteristics of the proposed structure are very sensitive to the thickness of the plasma medium within a certain frequency band for which the permittivity and permeability of LHM have negative values simultaneously.

Figure $2 \mathrm{~b}$ shows the dependence of effective wave in$\operatorname{dex} \beta$ on the propagation frequency $\omega$ for different values of the number density $n_{0}$ of the plasma medium i.e. $n=10^{17} \mathrm{~m}^{-3}, n=5 \times 10^{19} \mathrm{~m}^{-3}$, and $n=10^{21} \mathrm{~m}^{-3}$, with $F=0.56$ and $d=10^{-4} \mathrm{~m}$. Here we observe that, at some higher number density e.g. $n=5 \times 10^{19} \mathrm{~m}^{-3}$ or $n=10^{21} \mathrm{~m}^{-3}$, the lower region of propagation shifts upwards with negative slope (at smaller values of effective wave index $\beta$ ) whereas the upper region of propagation remains almost insensitive. Thus, for a certain range of the number densities (or dielectric constant $\varepsilon_{\mathrm{p}}$ of plasma medium), the upper region of propagation remains insensitive in the frequency band. It means for a given thickness $d=10^{-4} \mathrm{~m}$, our proposed structure has some flexibility for the values of number density (or dielectric constant of plasma medium). It is also noticed that at some higher values of number densities e.g. $n=10^{19} \mathrm{~m}^{-3}$ the propagation gap increases and then decreases with the increase of effective wave index. This shows that the propagation band or gap can be controlled by varying the number density.

Figure $2 c$ shows the dependence of effective wave in$\operatorname{dex} \beta$ on the propagation frequency $\omega$ for different values 


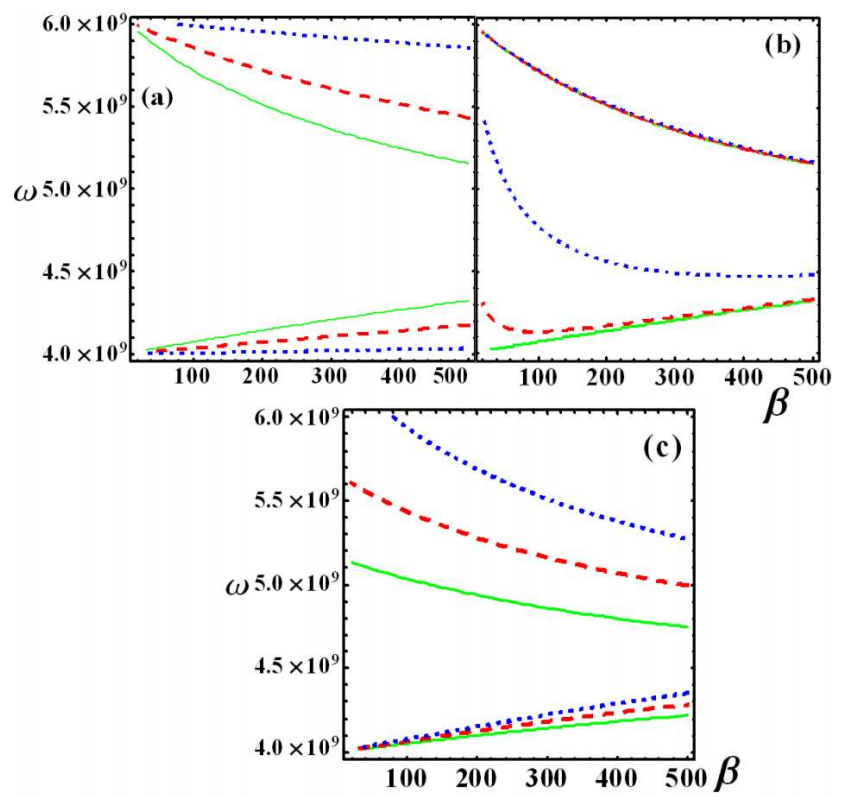

Fig. 2. A plot of effective wave index $\beta$ versus propagation frequency $\omega$ for a LHM/plasma/LHM structure. (a) For different thicknesses of the plasma medium i.e. $d=10^{-4} \mathrm{~m}(-), d=0.5 \times 10^{-4} \mathrm{~m}(--)$ and $d=0.1 \times 10^{-4} \mathrm{~m}(\ldots \ldots)$, with $F=0.56$ and $n=10^{17} \mathrm{~m}^{-3}$; (b) For different values of number densities i.e. $n=10^{17} \mathrm{~m}^{-3}(-), n=5 \times 10^{19} \mathrm{~m}^{-3}$ $(\ldots \ldots)$, and $n=10^{21} \mathrm{~m}^{-3}(\ldots \ldots)$, with $F=0.56$ and $d=10^{-4} \mathrm{~m}$; (c) For different values of filling factor i.e. $F=0.4(-), F=0.5(--)$ and $F=0.6$ $(\ldots \ldots)$, with $d=10^{-4} \mathrm{~m}$ and $n=10^{17} \mathrm{~m}^{-3}$.

of the filling factor $F$ of the LHM i.e. $F=0.4, F=0.5$ and $F=0.6$, with $d=10^{-4} \mathrm{~m}$ and $n=10^{17} \mathrm{~m}^{-3}$. We observe that the propagation gap increases with an increase of the filling factor $F$ of the split ring resonator in the LHM. This shows more flexibility in designing the split ring resonator of the LHM for the proposed structure.

\section{Conclusion}

The dispersion characteristics of TE surface waves have been investigated theoretically for a LHM/plasma/LHM structure. These investigations have been carried out in the specific frequency band for a LHM. For each thickness $d$ of the plasma medium, there are two propagation regions around a gap within the frequency band. In the upper region of propagation, the propagation frequency shows a decrease with the increase of effective refractive index. This negative slope indicates that our proposed structure behaves like LHM for upper region of propagation. Whereas, in the lower region of propagation, we observe an opposite trend. We have found that with the increase of effective wave index, this propagation gap in the frequency region decreases. We have also observed that as the thickness of the plasma medium decreases, the propagation gap in the frequency region increases. Further it is seen that the propagation characteristics are also sensitive to the number density of the plasma medium and it is noticed that the propagation band or gap can be controlled by varying the number density. Also, we have found that the propagation gap increases with the increase of the filling factor of the split ring resonator.

The present work is based on a simple model of a plasma-filled waveguide. Hence, some experimental work is definitely required in complementing the proposed theory developed in this study. Taking into account advantages of our plasma-filled waveguide over the other models applied in communication devices, it is evident that our model shows a band-gap structure which supports TE surface wave propagation in a narrow frequency interval where permittivity and permeability coexist with negative values. The main advantage of our proposed structure is that the propagation characteristics are not only dependent on the LHM parameters and thickness of the sandwich layer but also on the number density of the plasma medium. A plasma medium can have a significant effect if the plasma density is controlled and the geometry of the proposed structure is properly designed. This work may provide potential applications in the design of communication devices and components e.g. antennae, microstrips and waveguide couplers, etc., capable of supporting TE surface waves, with more flexibility on the thickness and number density of the plasma medium and also in the designing of the split ring resonator of the LHM.

\section{Acknowledgments}

We would like to thank the referee for his valuable comments.

\section{References}

[1] S.K. Ghosh, S.P. Pal, Czech. J. Phys. B 31, 25 (1981).

[2] R. Prasad, R. Prasad, A.R. Mantri, J. Phys (France) 51,1509 (1990).

[3] B. Zamir, R. Ali, Chin. Phys. B 20, 014102 (2011).

[4] J. Ma, H. Li, Q. Zhang, Y. Yin, X.-Z. Wang, Phys. Scr. 82, 015702 (2010).

[5] H.J. El-Khozondar, Z.I. Al-Sahhar, M.M. Shabat, Int. J. Electron. Commun. (AE Ü) 64, 1063 (2010).

[6] Q. Cheng, T.J. Cui, Appl. Phys. Lett. 87, 174102 (2005).

[7] S.-H. Liu, C.-H. Liang, W. Ding, L. Chen, W.-T. Pan, Prog. Electromagn. Res. 76, 467 (2007).

[8] B.-C. Liu, Y. Li, Z.-X. Lu, Chin. Phys. B 20, 037302 (2011).

[9] V.G. Veselago, Sov. Phys. Usp. 10, 509 (1968).

[10] W. Ding, L. Chen, C.H. Liang, Prog. Electromagn. Res. 70, 37 (2007).

[11] L. Chen, W. Ding, X.J. Dang, C.H. Liang, Prog. Electromagn. Res. 70, 257 (2007).

[12] R.A. Shelby, D.R. Smith, S. Schultz, Science 292, 77 (2001).

[13] J.B. Pendry, Phys. Rev. Lett. 85, 3966 (2000). 
[14] H.-C. Lin, J.-X. Pu, Chin. Phys. B 21, 054201 (2012).

[15] H. Xiong, J.-S. Hong, D.-L. Jin, Z.-M. Zhang, Chin. Phys. B 21, 094101 (2012).

[16] A.I. Ass'ad, H.S. Ashour, Adv. Condens. Matter Phys. 2009, ID 867638 (2009).

[17] A.I. Ass'ad, H.S. Ashour, Turk. J. Phys. 36, 207 (2012).

[18] H.S. Ashour, J. Mod. Phys. 4, 1165 (2013).

[19] Y. He, X. Zhang, Y.F. Yang, CF. Li, Chin. Opt. Lett. 9, 052301 (2011).

[20] Z.J. Wang, J.F. Dong, Prog. Electromagn. Res. 62 203 (2006).

[21] V.O. Girka, Y.P. Sergey, Y.N. Oleksandr, Prog. Electromagn. Res. B 46, 159 (2013).

[22] P.K. Mukherjee, J. Appl. Phys. 48, 3134 (1977).

[23] A.K.T. Assis, P.H. Sakanaka, Rev. Brasil. Fis. 20, 356 (1990).
[24] S.M. Khalil, N.M. Mousa, J. Theor. Appl. Phys. 8, 111 (2014)

[25] N.A. Krall, A.W. Trivelpiece, Principles of Plasma Physics, McGraw-Hill, New York 1990.

[26] P. Markos, C.M. Soukoulis Opt. Expr. 7, 649 (2003).

[27] D.R. Smith, W.J. Padilla, D.C. Vier, S.C. NematNaseer, S. Schultz, Phys. Rev. Lett. 84, 4184 (2000).

[28] I.V. Shadrivov, A.A. Sukhorukov, Y.S. Kivshar, A.A. Zharov, A.D. Boardman, P. Egan, Phys. Rev. E 69, 0166171 (2004).

[29] I.V. Shadrivov, A.A. Sukhorukov, Y.S. Kivshar, Phys. Rev. E 67, 057602 (2003).

[30] R. Ruppin, J. Phys. Condens. Matter 13, 1811 (2001).

[31] M.S. Hamada, A.I. Ass'ad, H.S. Ashour, M.M. Shabat, J. Microwav. Optoelectron. 5, 45 (2006). 